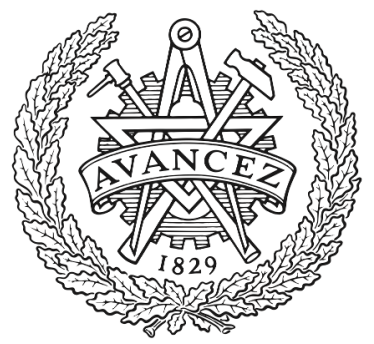

CHALMERS

UNIVERSITY OF TECHNOLOGY

\title{
Kinetics of oxidation of nm-sized soot spherules
}

Downloaded from: https://research.chalmers.se, 2023-04-26 04:23 UTC

Citation for the original published paper (version of record):

Zhdanov, V., Carlsson, P., Kasemo, B. (2008). Kinetics of oxidation of nm-sized soot spherules.

Chemical Physics Letters, 454: 341-44

N.B. When citing this work, cite the original published paper. 


\title{
Kinetics of oxidation of nm-sized soot spherules
}

\author{
Vladimir P. Zhdanov ${ }^{a, b, *}$, Per-Anders Carlsson ${ }^{a}$, Bengt Kasemo ${ }^{a}$ \\ ${ }^{a}$ Competence Center for Catalysis, Chalmers University of Technology, \\ S-412 96 Göteborg, Sweden \\ ${ }^{b}$ Boreskov Institute of Catalysis, Russian Academy of Sciences, \\ Novosibirsk 630090, Russia
}

\begin{abstract}
We analyse the non-catalytic soot oxidation kinetics predicted by four models implying, respectively, (i) monosized uniform spherically shaped soot spherules, (ii) monosized disk-like spherules formed of carbon sheets, (iii) spherically shaped spherules with a realistic size distribution, and (iv) disk-like spherules with a realistic size distribution. In all the cases, the reaction front is considered to move from the periphery to the center. The effect of the spherule size distribution on the oxidation kinetics is found to be appreciable and accordingly should be taken into account in interpretations of the corresponding experiments. The latter is illustrated by scrutinizing relevant experimental data and illustrating that model (iv) appears to be preferable.

Keywords: Models of surface kinetics; Oxidation; Reaction front; Soot structure; Nm-sized particles; Carbon sheets; Size distribution.

PACS numbers: 68.35.Fx, 82.65.+r

${ }^{*}$ Corresponding author: V.P. Zhdanov

E-mail address: zhdanov@catalysis.ru
\end{abstract}


Soot, produced at temperatures between 1600 and $1700 \mathrm{~K}$ or above during combustion of carbonaceous fuel, is an unwelcome product of industrial development. The effect of soot on health is harmful. For this reason, the formation of soot and its removal via oxidation (to $\mathrm{CO}_{2}$ and $\mathrm{CO}$ ) have long attracted attention of researchers working in different areas of applied sciences (for a review, see Refs. [1, 2, 3]). Now, the corresponding studies have got a new impulse due to the legislation-driven need to improve cleaning the exhaust gas of diesel and gasoline engines $[4,5]$. The formation and removal of soot are also of interest from the point of view of academic studies, because the corresponding rate processes and kinetics nicely illustrate many basic concepts of chemical kinetics (see e.g. numerous kinetic models $[6,7,8,9,10]$ describing various aspects of soot formation). Non-catalytic oxidation of soot usually occurs at temperatures above $550 \mathrm{~K}$. From the point of view of applications, this process is not sufficiently rapid at relatively low temperatures and accordingly should often be catalyzed [2].

Chemically, soot represents primarily carbon $(\simeq 84 \%)$ in combination with hydrogen $(\simeq 1 \%)$, oxygen $(\simeq 10 \%)$, and tracks of other elements including e.g. nitrogen $(\simeq 0.25 \%)$ and sulphur $(\simeq 1 \%)$ [1]. Structurally, soot is a heterogeneous aggregate exhibiting a few size scales [1]. Specifically, it is formed of "agglomerates" on the 100- $\mu \mathrm{m}$ scale. In turn, the agglomerates are composed of "particles" (0.1-1 $\mu \mathrm{m})$, which are a collection of "spherules" (10-70 nm). Agglomerates and particles are highly porous. For this reason, during soot oxidation, oxygen can easily reach spherules. Thus, basically, the soot oxidation is reduced to oxidation of spherules.

The structure of spherules is anisotropic. Specifically, a spherule can be viewed as a disk-like pellet formed of carbon sheets. The carbon atoms located inside such sheets are low reactive due to strong saturated carbon-carbon bonds. For this reason, the non-catalytic oxidation of soot is usually believed to start on the periphery of sheets and then the reaction front moves towards a sheet center (this assumption 
makes sense at least as a starting point for the analysis). The corresponding 2D kinetic models focused on oxidation of a single carbon sheet were proposed and analysed in detail in several articles $[11,12,13,14]$ by employing primarily the Monte Carlo technique. In such models, the conversion of each peripheral carbon atom is performed with the prescribed probabilities depending on the arrangement of adjacent carbon atoms. The recent DFT calculations $[15,16]$ seem to support the $2 \mathrm{D}$ scenario of soot oxidation. The detailed comparison of the results of calculations [11, $12,13,14]$ with the experimentally measured soot-oxidation kinetics were however not performed. One of the reasons of this state of the art is that the theoretical results were calculated numerically.

In experimental studies, the rate of non-catalytic soot oxidation is often described by employing empirical power-law equations with the Arrhenius dependence on temperature [17]. The apparent activation energy for this process is usually reported to be in the range from 140 to $170 \mathrm{~kJ} / \mathrm{mol}$ [1]. The reaction is found to be nearly first order in oxygen [1]. The reaction order in soot depends on conversion. At constant oxygen pressure, the latter dependence can be quantified by using the pseudo-first-order equation for the soot mass [1,3],

$$
d m / d t=-k m
$$

where $k$ is the conversion-dependent oxidation rate constant. Employing this strategy to fit their detailed kinetic data, Messerer et al. [3] conclude that the main stages of the soot-oxidation kinetics (for conversion above 0.25) are in agreement with the predictions of the 3D spherically symmetric uniform shrinking core model (for earlier applications of this model, see e.g. Refs. [18, 19] reviewed in [1]).

The goal of our Letter is to scrutinize the ways the experimental kinetic data should be compared with the theory. Specifically, we articulate that quantitative comparison of the experiment and theory makes sense only if one takes into account the size distribution of spherules (in the available experimental and theoretical works 
quoted above, this factor is ignored). Incorporating the spherule size distribution into the analysis, we show that for the interpretation of the most detailed experimental data [3] the 3D oxidation model implying 2D oxidation of sheets is in fact preferable compared to the conventional isotropic 3D shrinking core model.

To illustrate the likely dependences of $k$ on conversion, it is instructive first to recall how the spherically symmetric shrinking core model is used to describe oxidation of monosized spherules. In this model, each spherule is considered to be oxidized independently. This means that the contacts between spherules are neglected (this approximation seems to be reasonable, because the contact area is usually relatively small). The reaction front is assumed to propagate from the periphery to the center. In particular, the spherule radius decreases with increasing time as $r-v t$, where $r$ is the initial radius, and $v$ is the front-propagation velocity. The spherule volume shrinks as

$$
V=(4 \pi / 3)(r-v t)^{3}
$$

and accordingly

$$
d V / d t=-4 \pi v(r-v t)^{2} .
$$

Assuming all the spherules to be identical, the density of the spherules to be constant, and the spherule mass to be proportional to its volume, one gets [cf. Eq. (1)]

$$
k=-\frac{d V / d t}{V}=\frac{3 v}{r-v t} .
$$

The conversion is defined as

$$
\xi=1-\frac{V(t)}{V(0)}=1-\frac{(r-v t)^{3}}{r^{3}} .
$$

Employing this expression, one can rewrite Eq. (4) as

$$
k=\frac{3 v}{r(1-\xi)^{1 / 3}} .
$$


To compare with experiment or other models, it is convenient to use the ratio

$$
k / k_{0.5}=0.5^{1 / 3} /(1-\xi)^{1 / 3},
$$

where $k_{0.5}$ is the rate constant corresponding to $\xi=0.5$.

Let us now consider the case when a spherule is shaped as a disk-like pellet formed of carbon sheets so that the oxidation starts on the periphery of sheets and then the reaction front moves towards the center. For this scenario, we have [cf. Eqs. (2)-(7)]

$$
\begin{aligned}
& V=2 \pi h(r-v t)^{2}, \\
& d V / d t=-4 \pi v h(r-v t), \\
& k=2 v /(r-v t), \\
& \xi=1-(r-v t)^{2} / r^{2}, \\
& k / k_{0.5}=0.5^{1 / 2} /(1-\xi)^{1 / 2},
\end{aligned}
$$

where $r$ is the initial radius and $h$ is the thickness of a spherule.

Expressions (7) and (12) can be directly compared with experiment (in particular, expressions (7) was used in Ref. [3]). Their applicability is however expected to be limited, because the derivation does not take into account the spherule size distribution. According to numerous measurements (see e.g. Refs. [20, 21]), this distribution is rather broad. As already noted in the introduction, the spherule diameter is usually in the range from 10 to $70 \mathrm{~nm}$. The maximum of the distribution is at $30-40 \mathrm{~nm}$. The calculations [9] indicate that the size distribution may actually have a fine structure with a few narrow local maximums. For our present goals, the fine structure is not of interest, and taking into account typical experimental data (see e.g. Ref. [20]) we adopt the following distribution

$$
f(r)=\frac{4 r^{2}}{r_{m}^{3}} \exp \left(-\frac{2 r}{r_{m}}\right)
$$


where $r=r_{m}$ corresponds to the maximum.

With the spherule size distribution, we should operate with the average spherule volume, $V$, in order to describe the oxidation kinetics. For the spherically symmetric shrinking core model, for example, the average volume is given by [cf. Eq. (2)]

$$
V=(4 \pi / 3) \int_{v t}^{\infty}(r-v t)^{3} f(r) d r
$$

and accordingly

$$
d V / d t=-4 \pi v \int_{v t}^{\infty}(r-v t)^{2} f(r) d r
$$

Using these expressions, we obtain [cf. Eqs. (4) and (5)]

$$
\begin{aligned}
& k=\frac{3 v \int_{v t}^{\infty}(r-v t)^{2} f(r) d r}{\int_{v t}^{\infty}(r-v t)^{3} f(r) d r}, \\
& \xi=1-\frac{V(t)}{V(0)} .
\end{aligned}
$$

In expressions (14)-(16), the integration is performed from $r=v t$, because the spherules with $r<v t$ are completely converted to $\mathrm{CO}_{2}$ and $\mathrm{CO}$. Employing expressions (14)-(17) and distribution (13), one can easily calculate $k / k_{0.5}$ as a function of $\xi$.

For disk-like spherules formed of sheets, the size distribution should depend on $r$ and $h$. Usually, $h$ is comparable to $r$. As a reasonable approximation, we consider that $h$ is proportional to $r$, i.e., $h=\kappa r$, where $\kappa \simeq 1$ is a constant. In this case, the size distribution depends only on $r$ (in particular, one can use distribution (13)), and we have [cf. Eqs. (8)-(10)]

$$
\begin{aligned}
& V=2 \pi \kappa \int_{v t}^{\infty}(r-v t)^{2} r f(r) d r \\
& d V / d t=-4 \pi v \kappa \int_{v t}^{\infty}(r-v t) r f(r) d r \\
& k=\frac{2 v \int_{v t}^{\infty}(r-v t) r f(r) d r}{\int_{v t}^{\infty}(r-v t)^{2} r f(r) d r} .
\end{aligned}
$$


Above, we have presented the equations describing the soot oxidation kinetics in four cases. For monosized spherules, the results are analytical. For spherules with the size distribution, the kinetics can easily be calculated by using distribution (13). To compare the predicted dependences of $V, d V / d t$ and $k$ on time, it is convenient to normalize these variables to their values at $t=0$ and to normalize time to the time interval $t_{0.5}$ corresponding to $\xi=0.5$ as shown in Fig. 1. In all four cases, the dependence of $V$ on time is seen to be nearly the same at $t / t_{0.5} \leq 1.3$. On the later stages, the order of the kinetics (from fast to slow) is as follows: (i) monosized disk-like spherules [Eqs. (8)-(12)], (ii) monosized spherically shaped spherule [Eqs. (2)-(7)], (iii) disk-like spherules with the size distribution [Eqs. (18)-(20)], and (iv) spherically shaped spherules with the size distribution [Eqs. (14)-(17)].

The corresponding dependences of $k / k_{0.5}$ on $\xi$ are shown in Fig. 2 together with the experimental data obtained recently [3] for light-duty-vehicle diesel soot at $548 \leq$ $T \leq 723 \mathrm{~K}$. The experimentally measured dependence is seen to be qualitatively different compared to calculations at $\xi<0.15$. This effect is attributed [3] to rapid consumption of the most reactive soot components, accounting for $\simeq 15 \%$ of the initial soot carbon mass. For $\xi>0.15$, the experiment and calculations are in qualitative agreement. Quantitatively, the fit of the experiment is the best if one uses either the conventional 3D model with monosized spherically shaped spherules [Eqs. (2)-(7)] or the model implying disk-like spherules with the distribution of sizes [Eqs. (18)-(20)]. In these two cases, the agreement between experiment and calculations is reasonably good for $0.15 \leq \xi \leq 0.75$. For $\xi>0.75$, the agreement is slightly worse in both cases. Specifically, the former model somewhat overestimates $k / k_{0.5}$ while the latter model somewhat underestimates $k / k_{0.5}$. Physically, the latter model is obviously preferable because it takes into account the size distribution of spherules. This conclusion, drawn in relation with the results reported in Ref. [3], is also applicable to earlier studies (see e.g. Refs. [18, 19]) where the conventional 
3D shrinking core model was found to fit the experimental data.

Finally, it is instructive to illustrate the sensitivity of our conclusions to the assumptions used or, more specifically, to the shape of the size distribution of spherules. The results shown in Figs. 1 and 2 were obtained by employing distribution (13). An equally reasonable distribution is

$$
f(r)=\frac{27 r^{3}}{2 r_{m}^{4}} \exp \left(-\frac{3 r}{r_{m}}\right)
$$

Comparing the dependence of $k / k_{0.5}$ on $\xi$ for these two distributions (Fig. 3) indicates that the variation of the size distribution in the reasonable range does not change the conclusions. [Note that for disk-like spherules, the model with distribution (21) fits the experiment (Fig. 2) somewhat better than in the case of distribution (13).]

In summary, we have shown that the interpretation of experimentally measured kinetics of soot oxidation should take into account the size distribution of nm-sized soot spherules. With this distribution, the 3D model implying 2D sheet oxidation appears to be preferable compared to the conventional 3D shrinking core model.

\section{Acknowledgements}

This work has been supported by EMFO and the Competence Centre for Catalysis, which is hosted by Chalmers University of Technology and financially supported by the Swedish Energy Agency and the member companies AB Volvo, Volvo Car Corporation, Scania CV AB, GM Powertrain Sweden AB, Haldor Topsøe A/S, and The Swedish Space Agency. 


\section{References}

[1] B.R. Stanmore, J.F. Brilhac, P. Gilot, Carbon 39 (2001) 2247.

[2] B.A.A.L. van Setten, M. Makkee, J.A. Moulijn, Catal. Rev. Sci. Eng. 43 (2001) 489.

[3] A. Messerer, R. Niessner, U. Pöschl, Carbon 44 (2006) 307.

[4] E. Cauda, D. Fino, G. Saracco, V. Specchia, Chem. Eng. Sci. 62 (2007) 5182.

[5] N. Nejar, M. Makkee, M.J. Illan-Gomez, Appl. Catal. B: Environm. 75 (2007) 11.

[6] N.M. Marinov, W.J. Pitz, C.K. Westbrook, A.M. Vincitore, M.J. Castaldi, S.M. Senkan, C.F. Melius, Comb. Flame 114 (1998) 192.

[7] J. Appel, H. Bockhorn, M. Frenklach, Comb. Flame 121 (2000) 122.

[8] P. Dagaut, Phys. Chem. Chem. Phys. 4 (2002) 2079.

[9] J. Boulanger, F.S. Liu, W.S. Neill, G.J. Smallwood, J. Eng. Gas Turb. Pow.Tras. ASME 129 (2007) 877.

[10] R.I.A. Patterson, M. Kraft, Comb. Flame 151 (2007) 160.

[11] E.F. Brown, Comp. Chem. 12 (1988) 27.

[12] T. Kyotani, K. Ito, A. Tomita, L.R. Radovic, AICHE J. 42 (1996) 2303.

[13] F. Stevens, T.P. Beebe, Comp. Chem. 23 (1999) 175.

[14] W.Y. Chen, A. Kulkarni, J.L. Milum, L.T. Fan, AICHE J. 45 (1999) 2557.

[15] K. Sendt, B.S. Haynes, J. Phys. Chem. C 111 (2007) 5465. 
[16] J.M. Jones, D.H. Jones, Carbon 45 (2007) 677.

[17] P. Darcy, P. Da Costa, H. Mellottee, J.M. Trichard, G. Djega-Mariadassou, Catal. Today 119 (2007) 252.

[18] G. de Soete, in: Western States Section Meeting, Sant Lake City, Combustion Institute, 1988.

[19] J.P.A. Neeft, T.X. Nijhuis, E. Smakman, M. Makkee, J.A. Moulijn, Fuel 76 (1997) 1129.

[20] N.K. Meyer, Z.D. Ristovski, Envir. Sci. Techn. 41 (2007) 7309.

[21] T. Rönkkö, A. Virtanen, J. Kannosto, J. Keskinen, M. Lappi, L. Pirjola, Envir. Sci. Techn. 41 (2007) 6384. 


\section{Figure captions}

Fig. 1. Variables (a) $V$, (b) $W(W \equiv-d V / d t)$ and (c) $k$ as a function of time for monosized spherically shaped spherules (thick solid lines), monosized disk-like spherules (thin solid lines), spherically shaped spherules with the distribution of sizes (thick dashed lines), and disk-like spherules with the size distribution (thin dashed lines). All the variables are normalized to their values at $t=0$. Time is normalized to $t_{0.5}$ (by definition, $V\left(t_{0.5}\right) / V(0)=0.5$ ). The insets on panels (a), (b) and (c) show, respectively, the $r$ distribution [Eq. (13)], the cross-section of a spherically shaped spherule, and a disk-like spherule (in the latter two cases, the peripheral region marked by black colour is located behind the reaction front mowing towards the center).

Fig. 2. Oxidation rate constant as a function of conversion for monosized spherically shaped spherules (thick solid line), monosized disk-like spherules (thin solid line), spherically shaped spherules with size distribution (13) (thick dashed line), and disk-like spherules with size distribution (13) (thin dashed line). The filled circles show the average (over temperature) experimental data obtained by using the results presented in Fig. 8 in Ref. [3]. The circle size characterizes the scattering of data points at $0.25 \leq \xi \leq 0.75$. At $\xi \leq 0.25$ and $\xi \geq 0.75$, the scattering of data points is somewhat larger than the circle size.

Fig. 3. Oxidation rate constant as a function of conversion for spherically shaped spherules with size distribution (13) (thick lines), disk-like spherules with size distribution (21) (thin line), spherically shaped spherules with size distribution (21) (thick dashed lines), disk-like spherules with size distribution (21) (thin dashed line). 


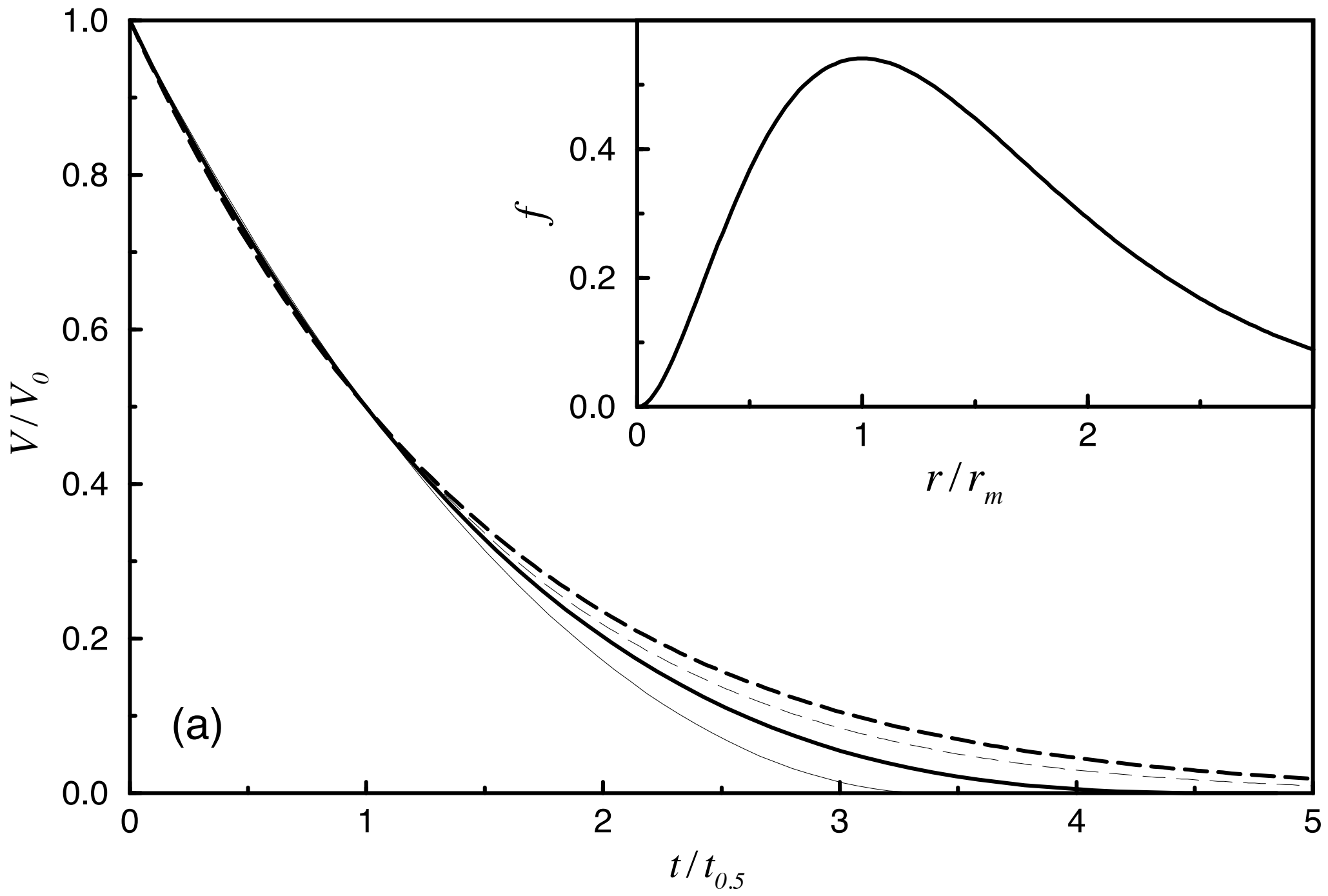

Fig. 1(a). 


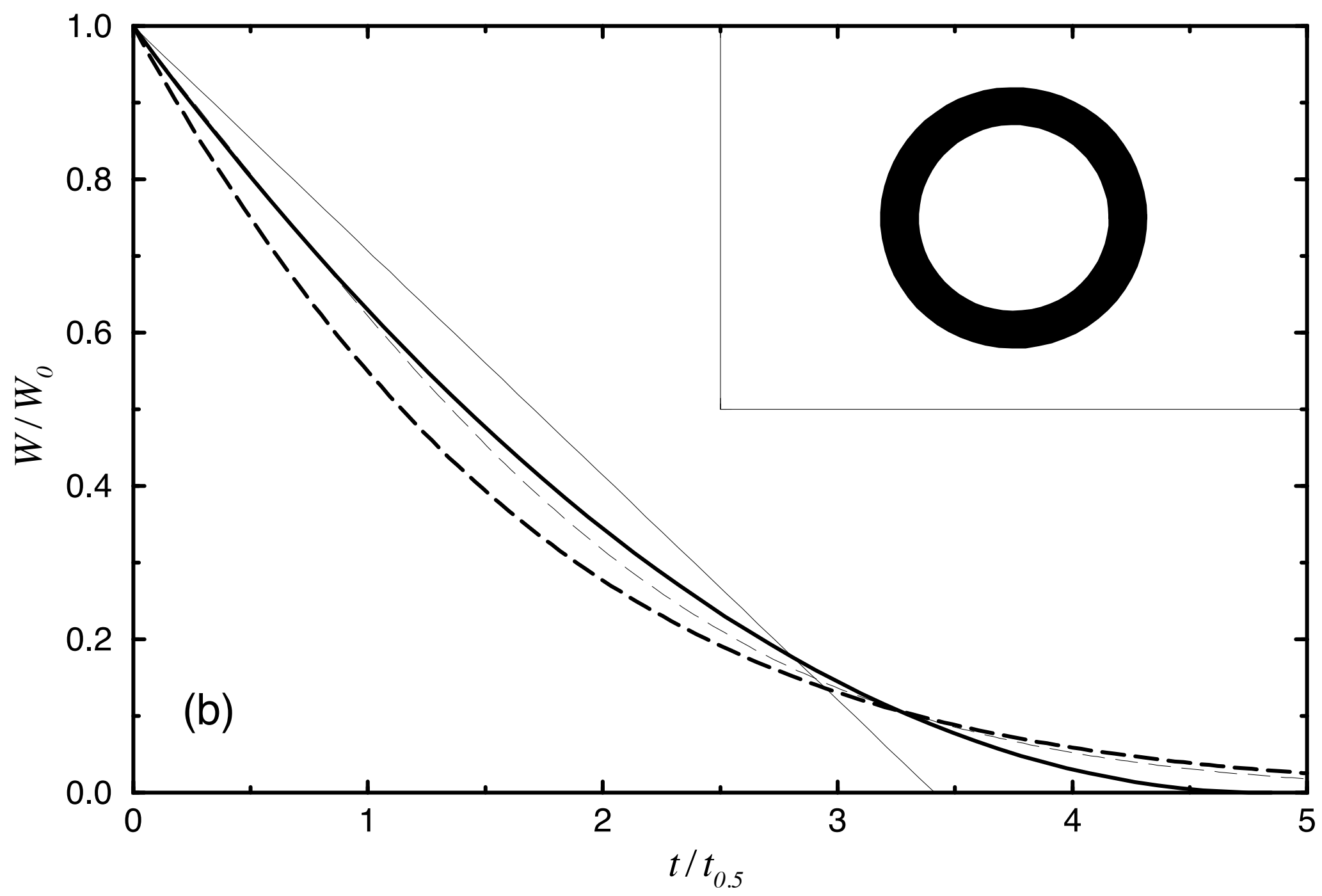

Fig. 1(b). 


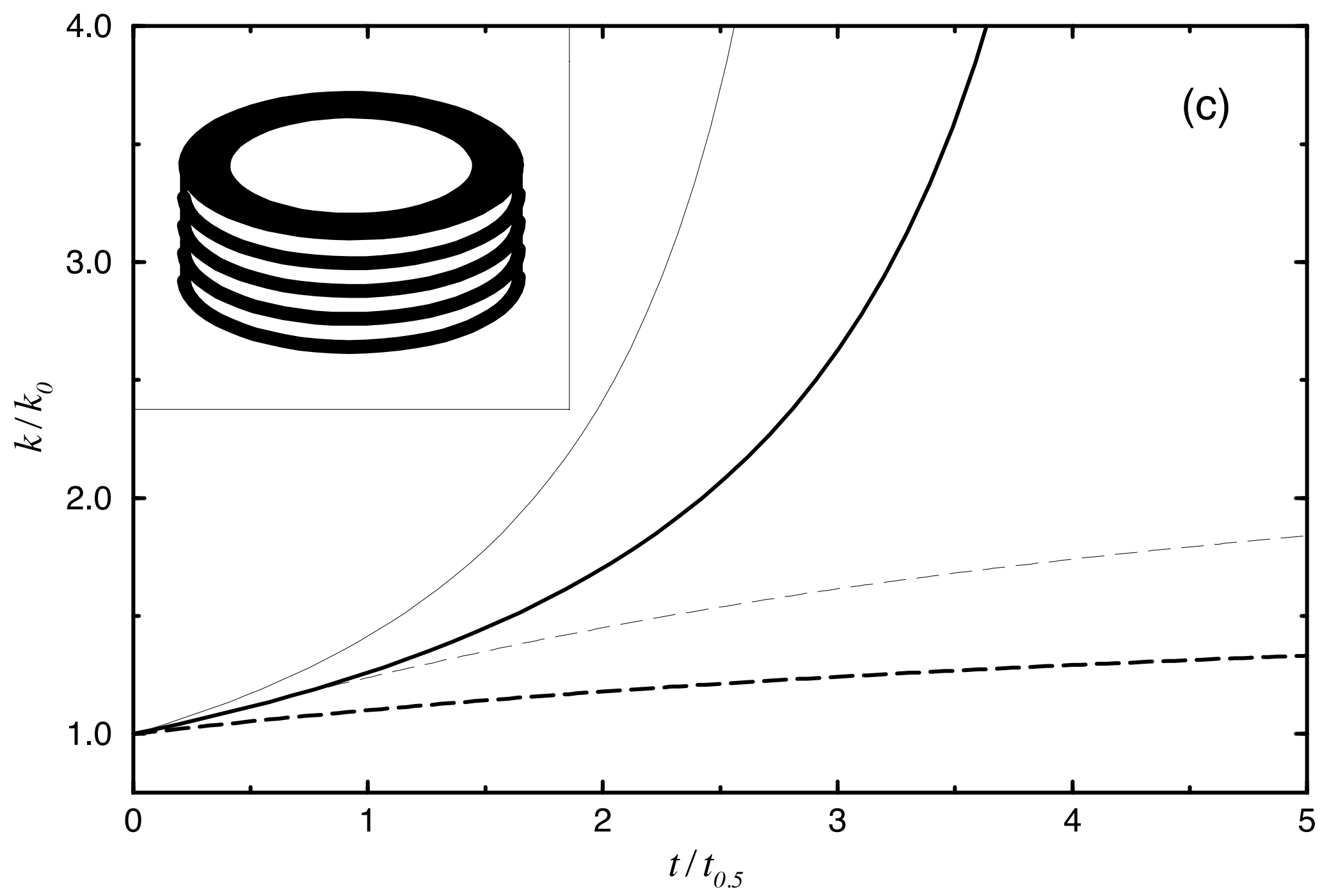

Fig. 1(c). 


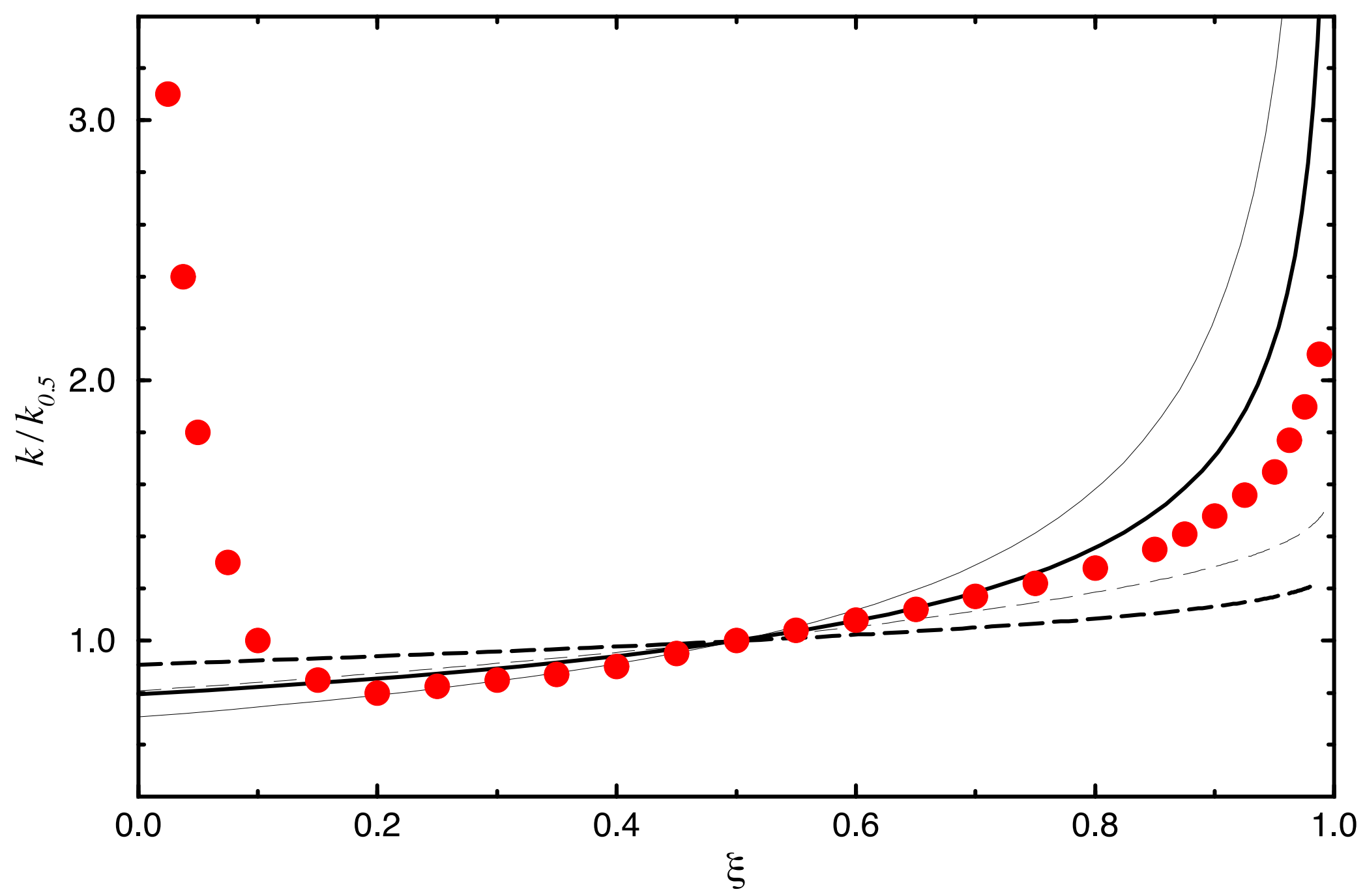

Fig. 2. 


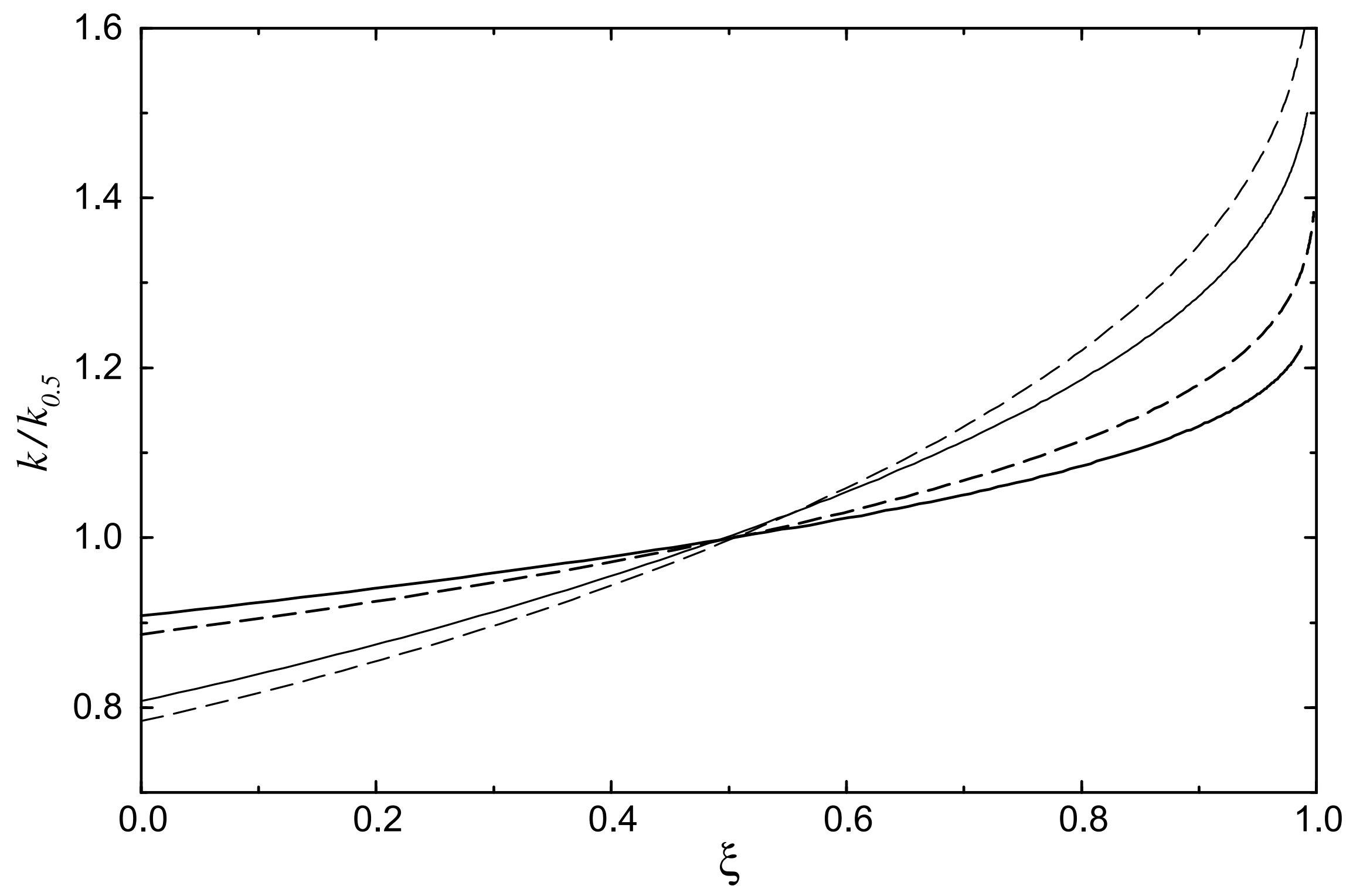

Fig. 3. 